\title{
Clodronate disodium for treatment of clinical signs of navicular disease - a double-blinded placebo-controlled clinical trial
}

\author{
Michael Frevel', Bradley L. King ${ }^{2}$, David S. Kolb ${ }^{3}$, Robert P. Boswell ${ }^{4}$, R. Stuart Shoemaker ${ }^{5}$, John C. Janicek 6 , Robert C. Cole?, \\ H. Melinda Poole ${ }^{8}$, and Susan L. Longhofer ${ }^{8}$ \\ 1 Bedburg, Germany \\ 2 King Veterinary Services, Gaston, IN, USA \\ 3 Lodi Veterinary Hospital, Lodi, WI, USA \\ ${ }^{4}$ Equine Sports Medicine and Diagnostic Imaging, Wellington, FL, USA \\ ${ }^{5}$ Idaho Equine Hospital, Nampa, ID, USA \\ ${ }^{6}$ Weems \& Stephens Equine Hospital, Aubrey, TX, USA \\ 7 Animal Imaging, Irving, TX, USA \\ 8 Dechra Development, Overland Park, KS, USA
}

\begin{abstract}
Summary: Navicular disease (also referred to as palmar foot syndrome or navicular syndrome, although not completely the same) causes lameness in many horses of all activity groups. The objective of this study was to compare clodronate efficacy at improving lameness grade in horses with clinical signs of navicular disease to that of horses given placebo. A randomized, multi-center, double-blinded clinical field study was conducted on 146 horses with forelimb lameness due to navicular disease. Navicular disease was confirmed by lameness examination (with diagnostic nerve blocks) and radiography of the affected distal forelimb(s). At study initiation, treated horses received $1.4 \mathrm{mg} / \mathrm{kg}$ clodronate disodium IM, and control (placebo) horses received a corresponding IM volume of $0.9 \%$ saline. Primary effectiveness was evaluated at Day 56 using a lameness grading scale, with positive response defined as improvement by at least 1 grade in the limb with the most apparent lameness, with no worsening of the lameness grade (compared to baseline) in the contralateral forelimb. Positive response rates at Days 28 and 180 were also recorded. On Day 56, 75\% of horses in the clodronate group were considered showing a positive response, versus only $3 \%$ in the placebo group $(p=0.003)$. Of the 86 horses treated with clodronate, 8 horses had an improvement of 3 lameness grades, 45 horses improved by 2 lameness grades and 16 horses improved by one lameness grade. The clodronate positive response rate remained high through Day 180 (65\%). Adverse events (primarily abdominal pain) were generally few, mild, and transient. In this study, clodronate disodium was demonstrated to be safe and effective in reducing the clinical signs of lameness associated with navicular syndrome.
\end{abstract}

Keywords: bisphosphonate, clodronate, clodronic acid, navicular syndrome, navicular disease, horse

Citation: Frevel M., King B. L., Kolb D. S., Boswell R. P., Shoemaker R. S., Janicek J. C., Cole R. C., Poole H. M., Longhofer S. L. (2017) Clodronate disodium for treatment of clinical signs of navicular disease: a double-blinded placebo-controlled clinical trial. Pferdeheilkunde 33, 271-279; DOI 10.21836/PEM20170308

Correspondence: Susan L. Longhofer DVM MS Dip. ACVIM, Dechra Development, 7015 College Blvd, Suite 510, Overland Park, KS, 66211,USA, e-mail: susan.longhofer@dechra.com

\section{Introduction}

Navicular disease (also referred to as palmar foot syndrome or navicular syndrome, although not completely the same) causes lameness in many horses of all activity groups. It usually presents as a chronic forelimb lameness, although cases with acute onset may also be seen. Navicular disease is a degenerative condition of the distal sesamoid (navicular) bone, and/or adjacent soft tissues structures such as the deep digital flexor tendon, the collateral sesamoidean ligaments, the distal sesamoidean impar ligament and the navicular bursa (Dyson et al. 2011). The exact cause and pathogenesis are unknown, but vascular compromise, abnormal biomechanical function and a genetic component may be implicated with a multifactorial etiology most likely (Pool et al. 1989, Dik et al. 2001, Dyson et al. 2011). Changes associated with bone remodeling have been well described, including bone resorption seen as radiolucent areas on radiographs, sclerosis of the flexor compact bone and spongiosa and entheseous new bone on the proximal border of the navicular bone (Dyson 2008, Verschooten et al. 1989, Pool et al. 1989). More recently, studies using MRI have further added to the understanding of the different changes that occur in the navicular bone as well as the surrounding soft tissues (Murray et al. 2006, Dyson et al. 201 1). Navicular disease cannot be readily cured, and treatment has focused on supportive therapies including rest, corrective shoeing, and anti-inflammatory medications (Schoonover et al. 2005, Belknap 2010). However, the association with bone loss has suggested that anti-resorptive agents such as bisphosphonates may be of benefit for the treatment of the osseus component of the disease (Denoix et al. 2003, Kamm et al. 2008). Bisphosphonates, as shown in experimental animal and human studies, act at several key places to inhibit osteoclast recruitment, adhesion, differentiation and resorptive activity; and to induce osteoclast apoptosis (Fleisch 2000, Russell 2007). Although circulatory half-lives are short, bisphosphonates accumulate rapidly in bone and remain there for long periods subject to skeletal turn over, so that active drug is liberated at the site of bone remodeling (Fleisch 2000).

Bisphosphonates have been used for decades to treat a variety of resorptive conditions in people (Scriba 2000). This class 
of drugs has also been evaluated as therapy for a variety of equine orthopedic problems, and the non-nitrogenous bisphosphonate tiludronate has been approved in several countries for treatment of navicular syndrome or bone spavin (Kamm et al. 2008).

Clodronate disodium, like tiludronate, is a non-nitrogenous bisphosphonate. It inhibits bone resorption by impeding the dissolution of hydroxyapatite crystals, as well as by direct cellular effects on osteoclasts (Fleisch 1991, Plosker and Goa 1994). Bisphosphonates have also been described to have anti-inflammatory and analgesic effects as shown in experimental animal model research and human in-vitro studies Plosker and Goa (1994) and Soto and Barbara (2014).

The purpose of the current study was to evaluate the efficacy and safety of clodronate for treatment of clinical signs associated with navicular disease in horses. This information was used to aid regulatory approval for an application as veterinary drug, so as to provide a new treatment option for horses suffering from this condition.

\section{Materials and Methods}

This multi-center, double blinded, placebo-controlled study was conducted under clinical conditions using client-owned horses examined by equine veterinarians in either the U.S. (5 investigation sites with 1 investigator each) or Germany (one investigator site with 1 investigator) in 2010-2011. No site was included in the statistical evaluation of effectiveness unless it had at least three evaluable clodronate treated horses and two evaluable placebo-treated controls.

Eligible horses were randomized to treatment groups of clodronate disodium [OSPHOS ${ }^{\circledR}$ Solution for Injection, Dechra Ltd., Skipton, North Yorkshire, United Kingdom] or saline control (placebo). Those in the treatment group received 1.4 $\mathrm{mg} / \mathrm{kg}$ clodronate disodium $(60 \mathrm{mg} / \mathrm{mL})$, equivalent to 1.19 $\mathrm{mg} / \mathrm{kg}$ clodronic acid. Placebo-treated horses received a corresponding volume of $0.9 \%$ saline $(2.33 \mathrm{~mL} / 100 \mathrm{~kg})$. Injections were administered IM in three sites on Day 0 . To ensure blinding of treatment group assignment, vials of clodronate or saline were coded with randomly assigned letter(s) (e.g. A to ZZZ) and were administered by a site treatment administrator, who was not the same person as the investigator.

To facilitate enrollment in this placebo-controlled trial, owners were offered the option of a compassionate use treatment of known clodronate at Day 56 even if deemed a treatment success. At the end of the study (Day 180), all remaining horses received a compassionate use treatment with clodronate.

\section{Study horses}

All were client-owned horses that were housed, fed and exercised according to individual owner preference. Lame horses of any breed were eligible for inclusion if they were cooperative with lameness examinations and otherwise clinically healthy. Medical records were evaluated and owners were interviewed for clinical histories. Horses were not eligible if there had been a change in shoe pattern within two weeks prior to the study, if lameness was not attributable to navicular disease or was potentially attributable to another condition, if there was evidence (scarring) or history of previous neurectomy, or if hematology or serum chemistries were abnormal.

Horses were excluded if they were pregnant, $<4$ years of age or had clinical signs of general illness (e.g. impaired general health, active systemic disease, loss of appetite, fever, etc.). Horses with hind limb lameness or evidence/history of musculoskeletal conditions that could have confounded the lameness exams were excluded. Horses were excluded if they had received extracorporeal shockwave therapy within 5 days of study initiation, NSAIDs during the 7 days prior to study initiation, short-acting corticosteroids 10 days prior to the study, or any long-acting corticosteroids, hyaluronic acid, isoxsuprine, pentoxifylline, or a systemically administered chondroprotective agent (i.e. PSGAGs) in the 30-day period before Day 0 . Oral chondroprotectives were allowed if treatment was initiated prior to Day 0. Radiographic exclusion criteria included signs indicative of lameness due to primary soft tissue injury, osteoarthritis, fractures or any condition with bony changes other than those related to navicular disease.

Diagnosis of navicular disease was based on a finding of unilateral or bilateral forelimb lameness (AAEP Lameness Scale Grade $\geq 2$ ), with clear reduction of lameness severity following anesthesia of the distal palmar digital nerves (at which time lameness may have shifted to the other limb). In bilateral lameness, the effectiveness evaluation was focused on the limb with the most apparent lameness. Enrolled horses were also required to have radiographic changes of the navicular bone, including at least one of the following degenerative changes: (1) >7 lucent zones (i.e. synovial invaginations) that were normal in shape, (2) at least one abnormally shaped lucent zone (traditionally known as lollipop-shaped areas) along the distal-horizontal or sloping borders of the navicular bone (i.e. Hallmark sign), (3) poor definition between the palmar cortex and the medulla due to subcortical sclerosis, (4) a crescent-shaped lucent zone in the central eminence of the flexor cortex of the navicular bone, (5) enthesiophyte formation on the proximal border of the navicular bone, (6) asymmetrically shaped navicular bones, or (7) proximal or distal extension of the flexor border of the navicular bone.

Ongoing/scheduled therapies (e.g. anthelmintics or vaccines) were continued except where these might interfere with study evaluation. Prohibited for 72 hours after the Day 0 treatment were medications such as aminoglycosides and tetracyclines that impact or are impacted by serum calcium levels. Administration of long-acting corticosteroids, systemically administered chondroprotective agents, or hyaluronic acid products was prohibited. NSAIDs or short-acting steroidal drugs were allowed only if deemed medically necessary, but all NSAIDs or short-acting steroids were to be withdrawn 7-10 days (respectively) before the scheduled effectiveness evaluations on Days 28, 56, and 180. Concurrent NSAID treatment did not influence the study results as described in the results section.

The pattern of shoeing and foot trimming for the front feet could not be changed during the period from two weeks prior to Day 0 until study termination. At the first visit, investigators recorded the shoeing pattern and confirmed the presence of heel wedges and/or rolled toes. At subsequent visits, investi- 
gator determined whether the shoeing pattern had changed and documented the date that the horse was last shod.

Horses continued their accustomed exercise regimen, as determined by the owner and based on the comfort level of the horse. Owners were interviewed at the scheduled evaluations to determine whether the exercise program had been consistent and if the horse had been exercised appropriately. Horses with changes to the level of exercise (decrease/increase or type) were not terminated from the study solely due to this change in exercise. Several horses had both increases and decreases in exercise throughout the study. No horse was considered non-evaluable nor was terminated for the reason of a change (decrease/increase) in exercise.

\section{Assessments}

Complete physical examinations were performed on Days 0 , 28, 56, and 180 (as appropriate). Examinations included assessment of body weight (via girth tape), temperature, respiration rate, heart rate, appearance, attitude, activity, and appetite. Hematology and serum chemistry samples were obtained on Days 0, 28 and 56. For individual horses, lameness was assessed over time by the same blinded investigator using the $0-5$ scale developed by the American Association of Equine Practitioners (AAEP) prior to treatment (Day 0) and at Days 28, 56, and 180 [American Association of Equine Practitioners website 2015]. The lameness evaluation included examination at the walk and trot while traveling down a straight line and circling to both right and left. Horses were also lunged if this did not cause undue discomfort. Other causes of lower foot pain (e.g. hoof bruises, hoof cracks, laminitis) were excluded based on clinical examination and use of hoof testers.

The same blinded investigator who performed the initial lameness evaluation confirmed the diagnosis by reduction in lameness following anesthesia of the distal palmar digital nerves in the limb with the most apparent lameness. Level of lameness in both forelimbs was graded prior to the nerve block. In bilaterally lame horses with identical grades in both legs, the limb with most lameness was selected at the discretion of the investigator. If desired, nerve block of the other forelimb was permitted with reassessment and grading following this block.

The foot with the most apparent lameness was subjected to a navicular series of digital radiographs on Days 0 and 180. Lateromedial, dorsoproximal-palmarodistal oblique (Oxspring's projection), palmaroproximal-dorsodistal oblique (skyline projection), and horizontal dorsopalmar views were obtained for each horse. The foot was cleaned and packed with a moldable packing product to eliminate artifacts; removal of shoes was at the discretion of each investigator. All radiographs were reviewed by a single board-certified radiologist (R.C. Cole), with Day 0 films assessed for study eligibility, and Day 180 films categorized as worsened, unchanged, or improved over baseline. Day 56 radiographs were not obtained as it was deemed to be too short of a period to document any major changes in bony structures.

Following injection, horses were observed for approximately 30 minutes for signs of immediate adverse events, then for an additional 1.5 hours. Owners were asked to report any abnormalities observed over the course of the study. Owners or primary caregivers were asked to maintain a diary and were interviewed on Days 28, 56, 180, and 240 (at Day 240 if needed because of compassionate use clodronate treatment at Day 180).

Horses developing hind-limb lameness during the study were evaluated on a case-by-case basis, but no horses were terminated for this reason. All horses that received compassionate use treatment with known clodronate after the Day 56 efficacy evaluation were excluded from the Day 180 efficacy evaluation.

\section{Statistical analyses}

The individual horse served as the experimental unit. Clinically valid evaluable cases were included in the efficacy assessment, and all horses that had been administered clodronate or placebo were included in the safety analyses. All data were analyzed with a computer software program (SAS ${ }^{\circledR}$, Cary, NC, USA). Statistical significance was evaluated at $\alpha=0.05$ (two sided tests) unless otherwise indicated.

The results from a pilot study in 23 horses with diagnosed navicular disease (12 horses in the clodronate group, 11 horses in the placebo group), showed that the positive reponse rate in the clodronate group was around $90 \%$ while success in the placebo group was around 10\%. Given this expected effect size, an enrollment ratio of 3:1 was chosen using a minimum of 100 clodronate treated horses and a minimum of 33 placebo horses.

Percent positive response (with $95 \%$ confidence interval $[\mathrm{Cl}]$ ) at the Day 56 evaluation served as the primary effectiveness endpoint. Positive response was defined as improvement by at least 1 grade in the limb with the most apparent lameness, with no worsening of the lameness grade (compared to baseline) in the contralateral forelimb. Differences in positive response rates between the groups were assessed using a generalized linear mixed model appropriate for binary data [SAS/STAT 9.2 User's guide 2008]. Treatment group was included in the model as a fixed effect, with site and site-bytreatment interaction included as random effects. Because of the likelihood of high failure rates in the placebo group, the "residual" term was included as an additional random effect so as to guard against over-dispersion. Positive response rates were also calculated for Days 28 and 180, but statistical tests were not performed as the primary effectiveness endpoint was success rate at Day 56 and no control group was available at Day 180.

For safety data analysis, categorical outcomes and continuous outcomes with less than five discrete levels were evaluated using a generalized linear mixed model appropriate for repeated-measures ordinal data, assuming a multinomial distribution and a cumulative logit link [SAS/STAT 9.2 User's guide 2008]. Continuous outcomes and those measured multiple times during the study (i.e. hematology, serum chemistry, and physical exam) were evaluated by repeated-measures analysis of covariance. Treatment, visit, and treatmentby-visit interaction were included in the model as fixed effects, 
with site, site-by-treatment interaction, and site-by-treatmentby-visit interaction included as random effects. Pre-treatment values were included as covariates when available. Blood results from the US and German laboratories were analyzed separately. Safety outcomes were considered statistically significant at $\alpha=0.10$.

\section{Results}

Of the 211 horses evaluated, 146 met inclusion criteria and received treatment with either clodronate or placebo. Enrolled horses represented a wide variety of breeds, ranged from 4 to 22 years of age, and weighed 367 to $601 \mathrm{~kg}$. There were 101 geldings, 41 mares and 4 stallions enrolled. Breeds included: 72 American Quarter Horses, 12 Rheinländer, 8
American Paint Horses, 5 each of Belgian Warmblood, Dutch Warmblood, Hanoverian, Sachsen Anhaltiner, Thoroughbred and Westfale, 4 Holsteiner, 3 each of Oldenburg and Selle Français and 1 each of American Quarterhorse/Morgan mix, Appaloosa, Warmblood, Belgian Sportshorse, Hessian Warmblood, Irish Horse, Latvian Warmblood, Swedish Warmblood, Bavarian Warmblood, Selle Luxembourgeois and Württemberger. Breed was unknown for three horses.

AAEP lameness grades were similar between groups (Grade 2, $66 \%$ and $71 \%$, and Grade 3, 34\% and 29\%, respectively for clodronate and placebo (Table 1) and radiographic findings between groups also were similar (Table 2). The preenrollment duration of the forelimb lameness was highly variable in both groups. Average lameness duration was 445 days (SD \pm 309 days) in the clodronate group and 321 days

\begin{tabular}{|c|c|c|c|c|}
\hline & Grade 0 & Grade 1 & Grade 2 & Grade 3 \\
\hline \multicolumn{5}{|l|}{ Day 0} \\
\hline Clodronate $(n=1111)$ & & & $73(66 \%)$ & $38(34 \%)$ \\
\hline Placebo $(n=35)$ & & & 25 (71\%) & $10(29 \%)$ \\
\hline \multicolumn{5}{|l|}{ Day 28} \\
\hline Clodronate $(n=89)$ & $19(21 \%)$ & $40(45 \%)$ & $25(28 \%)$ & $5(6 \%)$ \\
\hline Placebo $(n=29)$ & $1(3 \%)$ & $8(28 \%)$ & $13(45 \%)$ & $7(24 \%)$ \\
\hline \multicolumn{5}{|l|}{ Day 56} \\
\hline Clodronate $(n=86)$ & $41(48 \%)$ & $25(29 \%)$ & $16(19 \%)$ & $4(5 \%)$ \\
\hline Placebo $(n=28)$ & & & $20(71 \%)$ & $8(29 \%)$ \\
\hline \multicolumn{5}{|l|}{ Day 180} \\
\hline Clodronate $(n=61)$ & $25(41 \%)$ & $23(38 \%)$ & $12(20 \%)$ & $1(1 \%)$ \\
\hline Placebo $\left(n=N A^{a}\right)$ & & & & \\
\hline
\end{tabular}

Table 2 Radiographic findings at enrollment by treatment group and lameness score

\begin{tabular}{|c|c|c|c|c|c|c|c|c|}
\hline Group & & $\begin{array}{l}\text { More than } 7 \\
\text { lucent zones } \\
\text { (synovial } \\
\text { invaginations) on } \\
\text { distal horizontal/ } \\
\text { sloping border }\end{array}$ & $\begin{array}{l}\text { At least } 1 \\
\text { abnormally } \\
\text { shaped } \\
\text { synovial } \\
\text { invagination } \\
\text { on distal } \\
\text { horizontal/ } \\
\text { sloping border }\end{array}$ & $\begin{array}{c}\text { Poor } \\
\text { definition } \\
\text { between the } \\
\text { palmar } \\
\text { cortex and } \\
\text { medulla due } \\
\text { to subcortical } \\
\text { sclerosis }\end{array}$ & $\begin{array}{l}\text { Crescent- } \\
\text { shaped lucent } \\
\text { zone in the } \\
\text { central } \\
\text { eminence } \\
\text { of the flexor } \\
\text { cortex }\end{array}$ & $\begin{array}{c}\text { Enthesiophyte } \\
\text { formation } \\
\text { on the proximal } \\
\text { border }\end{array}$ & $\begin{array}{l}\text { Asymmetry } \\
\text { of the } \\
\text { navicular } \\
\text { bone }\end{array}$ & $\begin{array}{c}\text { Proximal } \\
\text { or distal } \\
\text { extension } \\
\text { of the flexor } \\
\text { border }\end{array}$ \\
\hline \multirow{2}{*}{$\begin{array}{l}\text { Clodronate } \\
\text { Grade } 2^{a}\end{array}$} & $\mathrm{n}$ & 7 & 72 & 33 & 17 & 18 & 2 & 0 \\
\hline & $\%$ & $6.3 \%$ & $64.9 \%$ & $29.7 \%$ & $15.3 \%$ & $16.2 \%$ & $1.8 \%$ & \\
\hline \multirow{2}{*}{$\begin{array}{l}\text { Clodronate } \\
\text { Grade } 3^{a}\end{array}$} & $n$ & 3 & 37 & 24 & 12 & 13 & 0 & 0 \\
\hline & $\%$ & $2.7 \%$ & $33.3 \%$ & $21.6 \%$ & $10.8 \%$ & $11.7 \%$ & & \\
\hline \multirow{2}{*}{$\begin{array}{l}\text { Clodronate } \\
\text { Total }^{a}\end{array}$} & $\mathrm{n}$ & 10 & 109 & 57 & 29 & 31 & 2 & 0 \\
\hline & $\%$ & $9 \%$ & $98 \%$ & $51 \%$ & $26 \%$ & $28 \%$ & $2 \%$ & $0 \%$ \\
\hline \multirow{2}{*}{$\begin{array}{l}\text { Placebo } \\
\text { Grade } 2{ }^{b}\end{array}$} & $n$ & 3 & 25 & 14 & 4 & 8 & 0 & 1 \\
\hline & $\%$ & $8.6 \%$ & $71.4 \%$ & $40 \%$ & $11.4 \%$ & $22.9 \%$ & & $2.9 \%$ \\
\hline \multirow{2}{*}{$\begin{array}{l}\text { Placebo } \\
\text { Grade } 3^{\text {b }}\end{array}$} & $\mathrm{n}$ & 1 & 10 & 7 & 3 & 2 & 1 & 1 \\
\hline & $\%$ & $2.9 \%$ & $28.6 \%$ & $20 \%$ & $8.6 \%$ & $5.7 \%$ & $2.9 \%$ & $2.9 \%$ \\
\hline \multirow{2}{*}{$\begin{array}{l}\text { Placebo } \\
\text { Total }^{b}\end{array}$} & $\mathrm{n}$ & 4 & 35 & 21 & 7 & 10 & 1 & 2 \\
\hline & $\%$ & $11 \%$ & $100 \%$ & $60 \%$ & $20 \%$ & $29 \%$ & $3 \%$ & $6 \%$ \\
\hline
\end{tabular}


(SD \pm 266 days) in the placebo group, with no significant difference between groups $(p>0.05)$. Prevalence of radiographic findings by baseline lameness score is shown in Table 2.

By the time of the primary-efficacy analysis (Day 56), 6 horses were excluded for the following reasons: injury (3), owner withdrawal (1), sold (1), failed to return (1). An additional 26 horses were excluded when two sites were removed from the statistical analysis due to insufficient number of placebo control horses. Due to compassionate use treatment with known clodronate after the Day 56 evaluation, there were no evaluable placebo-treated controls available at Day 180. Twentyfive placebo horses and 17 clodronate-treated horses received compassionate use treatment after the Day 56 evaluation. Of these, one placebo-treated horse and two clodronate treated horses had been graded as a "positive response". The owners of these horses opted for compassionate use treatment with hopes of observing additional improvement.

\section{Efficacy}

On Day 28, $67 \%$ of horses in the clodronate group were considered having a positive response versus $21 \%$ of those in the placebo group (Table 3). By the time of the primary-efficacy assessment (Day 56), the modelled positive response rate in the clodronate group had risen to $75 \%$, which was significantly higher $(p=0.003)$ than that in the placebo group (3\%). These Day-56 model rates are very similar to the crude values of $80 \%(69 / 86)$ for clodronate and $4 \%(1 / 28)$ for placebo. Among clodronate treated horses, Day-56 lameness grades had improved by one (16 horses), two (45 horses), or three ( 8 horses) grades as compared to the control group in which only one horse had an improved grade (Table 4).
Of the 68 clodronate treated horses that were deemed having a positive response on Day 56, 61 were evaluable at Day 180. Of these 61 horses, 52 remained showing a positive response at Day 180 based on improvement in lameness grade as compared to Day 0. However, 21 of these 61 evaluable horses demonstrated an increase in lameness grade at Day 180 as compared to their Day 56 evaluation. Including the 18 treatment failures at Day 56, the calculated overall positive response rate for clodronate solution at Day 180 is $65.8 \%(52 / 79)$. The modelled positive response rate in the clodronate group for Day 180 was $84 \%$ (Table 3).

A total of 59 clodronate-treated horses were assessed radiographically on Day 180. Of these, three (5\%) showed evidence of improvement, with the remainder unchanged over baseline.

The majority of the NSAIDs were administered after the Day 56 efficacy evaluation; there were only 4 instances of phenylbutazone and 1 instance of firocoxib administration from Days $0-56$. The results from these 5 horses were excluded from the efficacy analysis, thus NSAID administration did not complicate the study results at Day 56. Of the 9 horses that received NSAIDs after the Day 56 visit, only one was evaluable at Day 180, and the NSAID (phenylbutazone) had been discontinued for greater than 7 days prior to the Day 180 evaluation.

Safety

All 146 enrolled horses were included in the safety assessment. None were terminated from the study as a result of adverse events related to treatment.

\begin{tabular}{|c|c|c|c|c|c|c|c|c|c|c|c|c|}
\hline \multirow[t]{3}{*}{ Table 3} & \multicolumn{12}{|c|}{ Positive response ( $95 \%$ confidence interval) during the study ${ }^{a}$} \\
\hline & \multicolumn{5}{|c|}{ Number of horses (n) } & \multicolumn{7}{|c|}{ Positive response $(\%)(95 \% \mathrm{Cl})$} \\
\hline & \multicolumn{2}{|c|}{ Clodronate } & \multicolumn{3}{|c|}{ Placebo } & \multicolumn{3}{|c|}{ Clodronate } & \multicolumn{4}{|c|}{ Placebo } \\
\hline Day 28 & \multicolumn{2}{|c|}{89} & \multicolumn{2}{|r|}{29} & & \multicolumn{3}{|c|}{$67 \%(58 \%-77 \%)$} & \multicolumn{4}{|c|}{$21 \%(6 \%-35 \%)$} \\
\hline Day 56 & \multicolumn{2}{|c|}{86} & \multicolumn{3}{|c|}{28} & \multicolumn{3}{|c|}{$75 \%^{\mathrm{b}}(35 \%-94 \%)$} & \multicolumn{4}{|c|}{$3 \%(0.3 \%-26 \%)$} \\
\hline Day 180 & \multicolumn{2}{|c|}{61} & \multicolumn{3}{|c|}{$N A^{c}$} & \multicolumn{3}{|c|}{$84 \%(74 \%-93 \%)$} & \multicolumn{4}{|c|}{$N A^{c}$} \\
\hline \multicolumn{13}{|c|}{$\begin{array}{l}\text { a least-square means and } 95 \% \mathrm{Cl} \text { from the generalized linear mixed model. }{ }^{b} \text { significantly different }(p=0.003) \text { from placebo } \\
{ }^{c} \text { Not applicable - no evaluable controls due to compassionate use treatment after Day } 56\end{array}$} \\
\hline \multirow[t]{4}{*}{ Table 4} & \multicolumn{12}{|c|}{ Change in AAEP lameness grade at Day 28, 56 and 180 / Veränderung der Lahmheitsgrade nach AAEP am Tag 28,56 und 180} \\
\hline & \multicolumn{4}{|c|}{ Day 28} & \multicolumn{4}{|c|}{ Day 56} & \multicolumn{4}{|c|}{ Day 180} \\
\hline & \multicolumn{2}{|c|}{ Clodronate } & \multicolumn{2}{|c|}{ Placebo } & \multicolumn{2}{|c|}{ Clodronate } & \multicolumn{2}{|c|}{ Placebo } & \multicolumn{2}{|c|}{ Clodronate } & \multicolumn{2}{|c|}{ Placebo } \\
\hline & $\mathrm{n}$ & $\%$ & $\mathrm{n}$ & $\%$ & $\mathrm{n}$ & $\%$ & $\mathrm{n}$ & $\%$ & $\mathrm{n}$ & $\%$ & $\mathrm{n}$ & $\%$ \\
\hline $\begin{array}{l}\text { Improvement } \\
\text { by } 3 \text { grades }\end{array}$ & 4 & 4.5 & 0 & 0 & 8 & 9.3 & 0 & 0 & 5 & 8.2 & 0 & \\
\hline $\begin{array}{l}\text { Improvement } \\
\text { by } 2 \text { grades }\end{array}$ & 26 & 29.2 & 2 & 6.9 & 45 & 52.3 & 0 & 0 & 28 & 45.9 & 0 & \\
\hline $\begin{array}{l}\text { Improvement } \\
\text { by } 1 \text { grade }\end{array}$ & 34 & 38.2 & 8 & 27.5 & 16 & 18.6 & 1 & 3.6 & 20 & 32.8 & 0 & \\
\hline No change & 25 & 28.1 & 18 & 62.0 & 16 & 18.6 & 26 & 92.9 & 8 & 13.1 & 0 & \\
\hline $\begin{array}{l}\text { Worsened } \\
\text { by } 1 \text { grade }\end{array}$ & 0 & 0 & 1 & 3.4 & 1 & 1.2 & 1 & 3.6 & 0 & 0 & 0 & \\
\hline Total horses & 89 & & 29 & & 86 & & 28 & & 61 & & 0 & \\
\hline
\end{tabular}


Between Days 0 and 56, adverse events were reported for 30 horses $(27 \%)$ in the clodronate group and 7 horses $(20 \%)$ receiving placebo. Those adverse events considered to have probable, possible, or unknown relationships to clodronate (definition as used in the Guidelines on Pharmacovigilance for Medicinal Products for Veterinary Use by the European Union (Union 2011)) included pawing and/or rolling (2), colic (2), head bobbing (2), injection-site swelling (2), hives (1), lameness (6), lip licking (3), nervousness (7), and yawning (3). All were considered to be mild and transient, which was also the case for the adverse events in the control group. Between Days 56 and 240, adverse events were recorded for 34 horses receiving clodronate, but none were considered related to treatment. There were no apparent or observed interactions between clodronate and concurrent medications administered during the course of the study.

Shortly after clodronate administration on Day 0, 10 horses appeared uncomfortable, nervous, or otherwise experiencing pain, agitation, or potential signs of mild colic. Eight of these 10 horses were hand-walked until clinical signs resolved (approximately 15 minutes), one resolved after receiving an anti-inflammatory for hives, and signs resolved in one without treatment. Four additional horses experienced licking, yawning, head shaking, and/or rolling following administration of clodronate, but these signs quickly resolved spontaneously in three cases, and were considered normal behavior for the fourth horse.

There was no significant $(p<0.10)$ change in body weight, temperature, respiratory rate, or heart rate associated with clodronate treatment. Clodronate treatment was not associated with any clinically significant change in hematology or serum chemistry at any of the time points measured in this study.

\section{Discussion}

Horses treated with clodronate had a significantly higher positive response rate than did horses receiving placebo. Improvement was apparent by Day 28, which is approximately one month sooner than reported for cases of navicular syndrome treated with IV tiludronate (Denoix et al. 2003). Two thirds of treated horses showed at least 6 months of improvement from a one-time IM clodronate dose. This long lasting effect is in accordance with the pharmacokinetic and pharmacodynamic characteristics of clodronate, which include a relatively rapid clearance from the blood and a long lasting incorporation into bone tissue of several months (Plosker and Goa 1994).

Traditional treatments for navicular syndrome revolve around therapies such as corrective shoeing and treatment with antiinflammatories and other drugs (Schoonover et al. 2005, Belknap 2010). In the current study, the improvement produced by clodronate was in the absence of any ancillary treatment that may have confounded the results. Long-acting antiinflammatory drugs were prohibited, as were shorter-acting agents administered near the time of study initiation. Shoeing was kept unchanged from two weeks before the initial assessment until study termination.

Diagnosis in the current study was achieved using blinded lameness evaluation, radiographs, and nerve blocks, rather than imaging techniques such as MRI or CT scan, or means of objective lameness evaluation. Objective lameness evaluation such as force-plate assessment or body-mounted inertial sensor based system could have increased the reliability of lameness evaluation, although in the current study individual horses were evaluated by the same investigator over time, thereby excluding inter-observer variation (Schoonover et al. 2005, Keegan et al. 2010. Keegan et al. 2013). The chosen diagnostics were considered to be both sufficient and cost effective, but may have increased the risk of including horses with soft tissue injury of the heel, and of tendon or ligament injury within the foot. However, any introduced misclassification would have been equal for treatment and control group (non-differential) given the random nature of the design (randomized based on order of presentation), thereby skewing results toward the null and reducing the power to detect a significant effect (Rothman and Greenland 2008). The clear and significant improvement noted in the current study suggests that such misclassification was slight and/or that the benefits of clodronate treatment may have been underestimated.

There were relatively few adverse events potentially related to clodronate treatment, and these were considered to be mild and transient. Most common were clinical signs related to abdominal discomfort or mild nervous system effects, including nervousness, yawning, lip-licking, head-bobbing, and pawing/rolling. The vast majority resolved quickly with no intervention or after hand walking. Swelling at the site of administration occurred in only two $(0.8 \%)$ of the 248 clodronate injections (experimental and compassionate use). Over the course of the study, treatment had no significant effect on parameters commonly associated with pain such as heart or respiratory rate. There were no signs of elevated temperature or demineralized fractures, as has been observed with some bisphosphonates (Fleisch 1991, Plosker and Goa 1994). These findings are consistent with the excellent safety record of clodronate use in people (Plosker and Goa 1994).

The current study results support IM clodronate as a well-tolerated and effective treatment for horses diagnosed with a forelimb lameness, a positive reaction to palmar digital nerve block and radiological changes in the navicular bone. However, the fact that the effectiveness evaluation in this study was only carried out by means of a subjective lameness examination at three points in time should be considered. Further studies are necessary, using objective techniques for the evaluation of both lameness and pain causing inflammatory processes, to evaluate the effectiveness of clodronate in lameness caused by navicular syndrome.

\section{Acknowledgements}

The authors thank Dr. Steve Radecki for the statistical analysis of study results; Ms. Hayley Farr, Dr. Beasley Mason and Karen Bond for assistance with the conduct of the study.

\section{Animal Welfare Statement}

In support of worldwide regulatory approvals, the study was designed and conducted according to Good Clinical Practi- 
ce, Guidance GL9, International Cooperation on Harmonization of Technical Requirements for Registration of Veterinary Medicinal Products. All study related procedures were conducted under best veterinary practices. Approvals to conduct the clinical trial were received from the applicable regulatory agencies in the U.S. and Germany.

\section{Informed consent statement}

Informed consent was obtained from each owner or authorized representative.

\section{Conflict of interest statement}

Financial support for this study was provided by Dechra Ltd, Skipton, North Yorkshire, United Kingdom. The information from this study was used to aid regulatory approval for an application as veterinary drug. The authors Poole and Longhofer were employed by Dechra at the time of the study.

\section{References}

American Association of Equine Practitioners website (2015) Lameness exams: evaluating the lame horse. Available at: www.aaep.org/ info/horse-health? publication $=836$. Accessed June 25, 2015

Belknap J. K. (2010) Lameness in horses: Disorders of the foot. In: Kahn CM, ed. The Merck Veterinary Manual. 10th ed. Whitehouse Station, NJ, USA: Merck \& Co, Inc, 997-1046

Denoix J. M., Thibaud D., Riccio B. (2003) Tiludronate as a new therapeutic agent in the treatment of navicular disease: a doubleblind placebo-controlled clinical trial. Equine Vet. J. 35, 407-413

Dik K., van den Belt A., Enzerink E., van Weeren P. (2001) The radiographic development of the distal and proximal double contours of the navicular bone on dorsoproximal-palmarodistal oblique (upright pedal) radiographs from age 1 to 11 months. Equine Vet. J. 33, 70-74

Dyson S. (2008) Radiological interpretation of the navicular bone. Equine Vet. Educ. 20, 268-280

Dyson S., Murray R., Schramme M., Blunden T. (2011) Current concepts of navicular disease. Equine Vet. Educ. 23, 27-39

Fleisch H. (1991) Bisphosphonates. Pharmacology and use in the treatment of tumor-induced hypercalcemia and metastatic bone disease. Drugs 42, 919-944
Fleisch H. (2000) Biological effects. Bisphosphonates in bone disease: from the laboratory to the patient. 4th ed. San Diego: Academic Press, 34-51

Kamm L., Mcllwraith W., Kawcak C. (2008) A review of the efficacy of tiludronate in the horse. J. Equine Vet. Sci. 28, 209-214

Keegan K. G., Dent E. V., Wilson D. A., Janicek J., Kramer A., Lacarrubba A., Walsh D. M., Cassells M. W., Esther T. M., Schiltz P., Frees K. E., Wilhite C. L., Clark J. M., Pollitt C. C., Shaw R., Norris $T$. (2010) Repeatability of subjective evaluation of lameness in horses. Equine Vet. J. 42, 92-97

Keegan K. G., Wilson D. A., Kramer J., Reed S.K., Yonezawa Y., Maki H., Pai P. F., Lopes M. A. (2013) Comparison of a body-mounted inertial sensor system-based method with sunjective evaluation for detection of lameness in horses. Am. J. Vet. Res. 74, 17-24

National Office of Animal Health (2014) Equidronate ${ }^{\circledR} 500$ mg Lyophilisate for solution for infusion. In: Compendium of data sheets for animal medicines. Middlesex, UK, National Office of Animal Health, 188-189

Murray R., Blunden A., Schramme M., Dyson S. (2006) MRI characterisitcs of the foot in horses with palmar foot pain and control horses. Vet. Radiol. Ultrasound 47, 1-16

Plosker G. L., Goa K. L. (1994) Clodronate. A review of its pharmacological properties and therapeutic efficacy in resorptive bone disease. Drugs 47, 945-982

Pool R. R., Meagher D. M., Stover S. M. (1989) Pathophysiology of navicular syndrome. Vet. Clin. North Am. Equine Pract. 5, $109-$ 129

Rothman K. J., Greenland S. (2008) Precision and validity in epidemiologic studies. In: Rothman K. J., Greenland S., Lash T. L., eds. Modern Epidemiology. 2nd ed. Philadelphia: Lippincott Williams \& Wilkins; 418-458

Russell R. G. (2007) Bisphosphonates: mode of action and pharmacology. Pediatrics 119 (Suppl 2), S150-S162

SAS/STAT 9.2 User's guide. Cary, NC (2008) SAS Institute Inc.; pages 2078-2419, 38864078

Schoonover M. J., Jann H. W., Blaik M. A. (2005) Quantitative comparison of three commonly used treatments for navicular syndrome in horses. Am. J. Vet. Res. 66, 1247-1251

Scriba G. K. E. (2000) Bisphosphonate im Überblick. Pharmazie in unserer Zeit 29, 50-56

Soto S. A., Barbara A. C. (2014) Bisphosphonates: Pharmacology and Clinical Approach to Their Use in Equine Osteoarticular Diseases. J. Equine Vet. Sci. 34, 727-737

Union E. (2011) Volume 9B of The Rules Governing Medicinal Products in the European Union, p. 165

Verschooten F., Roels J., Lampu P., Desmet P., De Moor A., Picavet T. (1989) Radiographic measurements form the lateromedial projection of the quine foot with navicular disease. Res. Vet. Sci. 46, 15-21 